\title{
Inhomogeneity Effects in HTS Coated Conductors Used as Resistive FCLs in Medium Voltage Grids
}

\author{
Daniele Colangelo ${ }^{1}$, Bertrand Dutoit ${ }^{1}$ \\ ${ }^{1}$ EPFL, École Polytechnique Fédérale de Lausanne, Lausanne 1015, Switzerland \\ E-mail: daniele.colangelo@epfl.ch
}

\begin{abstract}
.
For Resistive Fault Current Limiters (RFCLs) based on high temperature superconducting coated conductors (HTS-CCs), inhomogeneity, in terms of critical current and geometrical imperfections such as stabilizer and substrate thicknesses, plays a very important role and it may limit the penetration of such devices into the electrical market. This paper presents an electro-thermal model, developed in SimPowerSystem ${ }^{\mathrm{TM}}$, able to describe the transient respond of HTS-CC candidates with different degrees of inhomogeneity, both in terms of critical current and of thickness stabilizer. Critical current inhomogeneity has been modeled with Gaussian distributions. Layers thicknesses used in the simulations have been chosen by fitting the temperature dependence of real tape resistances. Our approach considers relative inhomogeneity positions as well as thermal conduction along the HTS-CC length. The model is tuned using experimental measurements made on $\mathrm{ReBaCuO}$ coated conductors. A new dynamical thermal calibration of the model is proposed using finite element method calculations. Inhomegeneity effects with different possible faults (e.g. three phases and single phase short-circuit) are presented.
\end{abstract}




\section{Introduction}

Resistive fault current limiters, based on $\mathrm{ReBaCuO}$ coated conductors (CCs), are promising devices to enhance the stability and the security of the electricity network [1], they can drastically reduce investments for grid upgrade. However, the transient respond of such device needs to be studied for each possible application. The RFCL limiting performance is directly affected by several parameters (e.g. line impedance, fault impedance, fault duration, electrical angle, nature of the load etc..) and a particular operating configuration may underline the $\mathrm{CC}$ weak points (e.g. inhomogeneities in term of critical current) damaging the device or leading it to an unexpected limitation. Therefore, it is important to perform consistent simulations able to predict RFCL limiting characteristics in the multiplicity scenarios the grid can present. In order to optimize the design of RFCLs for MV applications and to study their integration in the grid, we developed a numerical analysis software module using Simulink $\AA^{\circledR}$ SimPowerSystem ${ }^{\mathrm{TM}}$ library.

\section{Concept of the model}

Our research is valid for any $\mathrm{CC}$, concrete examples and calculations are made using specific characteristics of $\mathrm{ReBaCuO}$ CCs manufactured by SuperPower Inc. (SP) [2]. The tape architecture consists in silver stabilizer, silver over-layer, ReBaCuO layer, buffer and hastelloy C-276 substrate [3]. Each phase of the RFCL device is composed by several paralleled tapes to obtain the required total critical current $\left(I_{c, t o t}\right)$. In our analysis, we supposed a overcurrent of $20 \%$ the rated current of the line $\left(I_{n}\right)$, so $I_{c, t o t} \geq 1.2 \sqrt{2} I_{n}$. The longitudinal length of each paralleled tape $\left(L_{t o t}\right)$ is divided in $N_{b l o c k s}$ isothermal blocks of length $L_{k}=L_{\text {tot }} / N_{\text {blocks }}$ (figure $1 a$ ). The heat exchange between tapes and liquid nitrogen bath is not considered (adiabatic assumption) and the buffer layer effect is neglected. Each block implements the electrothermal properties described by the following equations.

\subsection{Electrical equations}

The electrical resistance of each block is given by

$$
\frac{1}{R_{k}}=\frac{1}{\rho_{s c_{k}}\left(T_{k}\right) \frac{L_{k}}{A_{s c}}}+\sum_{j=1}^{N-1} \frac{1}{\rho_{N s c_{j}}\left(T_{k}\right) \frac{L_{k}}{A_{j}}}, \quad j_{t h} \text { layer }
$$

where $\rho_{s c_{k}}$ is the superconducting layer (SC) resistivity and $\rho_{N s c_{j}}$ is the equivalent resistivity of all no-superconducting (noSC) materials that compose the tape architecture (e.g hastelloy $\mathrm{C}-276$ and silver). The $\mathrm{SC}$ cross-section area $A_{s c}$ is $1 \mu \mathrm{m}$ thickness and $12 \mathrm{~mm}$ width, while the noSC materials ones $\left(A_{j}\right)$ are discussed is section 
4. In particular, $\rho_{s c_{k}}$ is calculated by

$$
\rho_{s c_{k}}= \begin{cases}\rho_{p l_{k}}=\frac{E_{c}}{J_{c k}(T)}\left(\frac{|J|}{J_{c k}(T)}\right)^{\left(n_{k}-1\right)}, & T_{k}<T_{c} \\ \rho_{\text {norm }}(T) & T_{k} \geq T_{c} \text { or } \rho_{p l_{K}} \geq \rho_{\text {norm }}\end{cases}
$$

The SC transition to normal state is modeled with a particular form of the well known power law, where $E_{c}$ is a constant $(1 \mu \mathrm{V} / \mathrm{cm}), J$ is the current density through the $\mathrm{SC}$, whereas $J_{c k}$ and $n_{k}$ are respectively, the critical current density and the transition index of block $k$ (equation 3 and 4 ). The critical current density $J_{c k}$ corresponds to $I_{c k} / A_{s c}$. Parameters $\alpha$ and $\beta$ are constants used to describe the temperature dependence of $I_{c}$ and $n$. The $\mathrm{ReBaCuO}$ normal state resistivity $\rho_{\text {norm }}$ has been obtained from literature [4].

$$
\begin{gathered}
I_{c k}=I_{c 0 k}\left(\frac{T_{c}-T_{k}}{T_{c}-T_{L N 2}}\right)^{\alpha}, T_{k}<T_{c} \\
n_{k}=n_{0 k}\left(\frac{T_{c}-T_{k}}{T_{c}-T_{L N 2}}\right)^{\beta}, T_{k}<T_{c}
\end{gathered}
$$

The SC transition temperature $\left(T_{c}\right)$ corresponds to $91 K$ (see inset figure 2 ) whereas, $T_{k}$ is the temperature of the considered block and $T_{L N 2}$ is the operating temperature, generally the liquid nitrogen one $(77 \mathrm{~K})$. The tape inhomogeneity is modeled by assigning different $I_{c 0 k}$ and $n_{0 k}$ to the $k$-blocks (see section 5 ).

\subsection{Thermal equations}

The model solves the heat equation

$$
C_{p k} \frac{\partial T_{k}}{\partial t}=Q_{k}\left(T_{k}\right)
$$

where the heat capacity of block $k\left(C_{p k}\right)$ is reduced by the factor $L_{e q} / L_{k}$ and it is obtained by

$$
C_{p k}=\sum_{j=1}^{N} \varrho_{m j} c_{p j}\left(T_{k}\right) A_{j} L_{e q}, \quad j_{t h} \text { layer }
$$

where $\varrho_{m j}, c_{p_{j}}$ and $A_{j}$ are respectively the mass density, the specific heat capacity and the cross section area of the $j_{t h}$ layer of the $k_{t h}$ block, while $L_{e q}$ is the thermal length used for the calibration of the model $(5 \mathrm{~mm}$, see section 3$)$. The temperature gradient between the HTS-CC layers is neglected. The heat source, $Q_{k}$, is composed by two terms: the joule losses generated in block $k$ (reduced by the factor $L_{e q} / L_{k}$, see equation 8) and the heat exchange with block $k$-1 and block $k+1$ (equation 8).

$$
\begin{gathered}
Q_{k}=R t_{k}\left(I, I_{c k}, T\right) I^{2}+\left(\frac{T_{k-1}-T_{k}}{R_{T}}\right)+\left(\frac{T_{k+1}-T_{k}}{R_{T}}\right) \\
\frac{1}{R t_{k}}=\frac{1}{\rho_{s c_{k}}\left(T_{k}\right) \frac{L_{e q}}{A_{s c}}}+\sum_{j=1}^{N-1} \frac{1}{\rho_{N s c_{j}}\left(T_{k}\right) \frac{L_{e q}}{A_{j}}}, \quad j_{t h} \text { layer }
\end{gathered}
$$




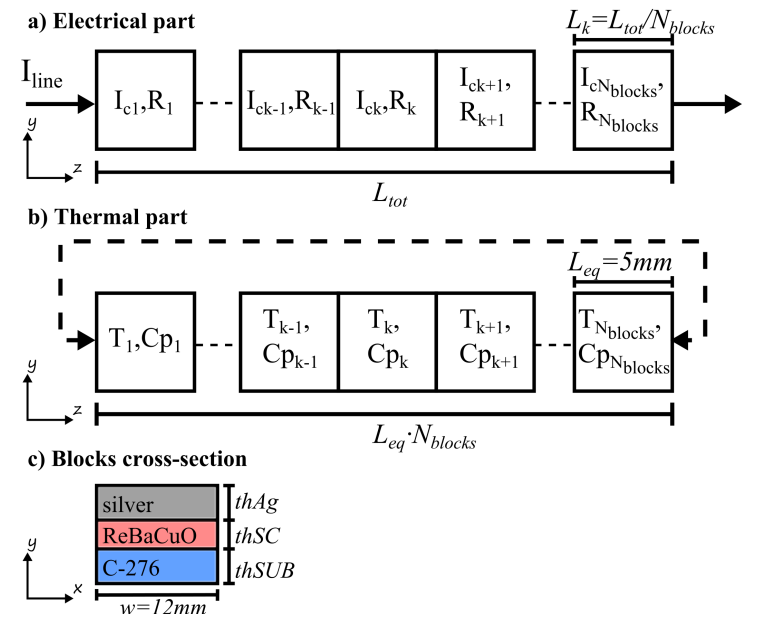

Figure 1: Electrical and thermal block connections. The electrical part consider the single tape length $L_{t o t}(a)$ whereas, the thermal one consider a portion of tape equal to $L_{e q} \cdot N_{\text {blocks }}(b)$. Each block implements the same cross-section $(c)$.

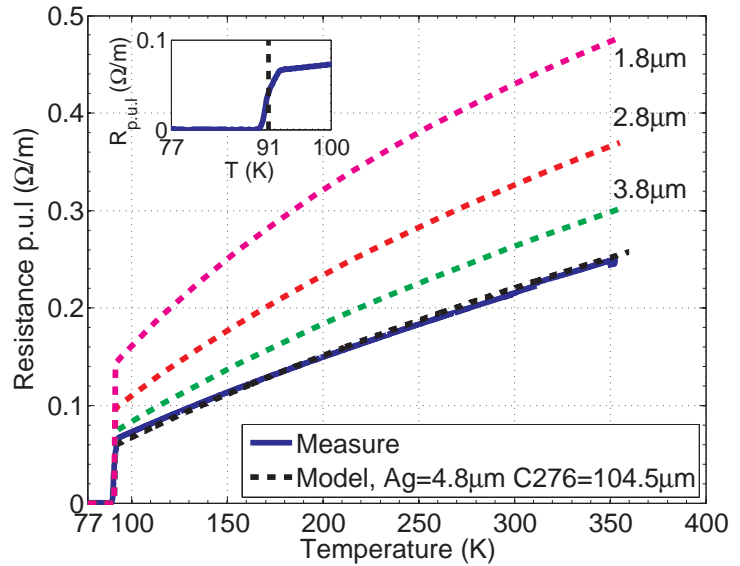

Figure 2: Resistance temperature dependence of the superconducting tape for $T>$ $T_{c}$. Fitted with simulink model (black dashed line) and $\mathrm{R}(\mathrm{T})$ predictions for three different silver stabilizer thicknesses (green, red and magenta dashed lines).

The thermal resistance $R_{T}$ is determined by comparison to fitted data obtained from finite element method calculations. In real applications $L_{t o t}$ is between 100 and $300 \mathrm{~m}$. Since $L_{e q}$ is $5 \mathrm{~mm}$, this implies a number of blocks larger than 20000. This number can be reduced, calculating the thermal conduction dynamics in a small part of the tape and assuming this part is periodically repeated along the whole tape length $L_{\text {tot }}$ (figure $1 b$ ). Therefore heat capacity and heat source have to be scaled by a factor $L_{e q} / L_{k}$.

\section{Dynamical calibration of the thermal part}

Comparing temperatures obtained by finite element method with ones calculated by the simulink model, it allows to calibrate $R_{T}$. Based on the same concept reported in a previous work [5] a thermal calibration has been performed. The finite element method model (FE) has been extended to 100 sectors, each one of length $L_{e q}=5 \mathrm{~mm}$. In previous calculations, each sector had different initial temperature and any power source was considered. Therefore, $R_{T}$ was chosen monitoring sectors temperature profiles. With the new method, all sectors have the same initial temperature $(77 \mathrm{~K})$ and in order to simulate the effect of $I_{c}$ inhomogeneity, a source of constant power density has been simulated in the SC layer of each sector. The simulink model has been extended to 100 blocks too. Power sources equivalent to the power source density of the FEM sectors, have been implemented on the simulink model blocks. The temperature evolution of the 100 sectors of the FEM has been compared with the corresponding block temperature $\left(T_{\text {sim }}\right)$ of the simulink model (only the first 5 sectors are drawn in figure $3 a$ and $3 b$, 
$T_{S A}, T_{S B}, T_{S C}, T_{S D}$ and $\left.T_{S E}\right)$. The power sources are respectively, $q 1=5 \cdot q_{r e f}, q 2=3.5 \cdot q_{r e f}$, $q 3=6 \cdot q_{r e f}, q 4=3 \cdot q_{\text {ref }}$ and $q 5=4 \cdot q_{\text {ref }}$ where, the power reference $\left(q_{\text {ref }}\right)$ is $3 \mathrm{~W}$ for figure $3 a$ and $q_{r e f}=8 W$ for figure $3 b$. In both cases the simulink model fits well the data obtained by FEM. As in the previous calibration, the temperature in the FE was monitored in the center of the the SC layer sectors. It is important to point out that our calibration method gives good results even when a low amount of power is dissipated. With high power sources (corresponding to high fault currents through the tape) the thermal conduction between different zones of the tape tends to be negligible compared to the case of low fault currents. Therefore, we are confident to assert that our calibration method can be considered valid for a wide rage of real operating conditions.

\section{Equivalent geometrical parameters}

Geometrical tape imperfections alter the performance of the RFCL device. For instance, imperfections of the C-276 substrate influence the total heat capacity and so the maximum energy the tape can withstand. The total silver stabilization (silver stabilizer + silver over-layer) conditions the current sharing between layers, hence, the impedance that the tape presents to the circuit during its limiting phase. The substrate and stabilizer thicknesses implemented in the model, have been determined fitting the resistance temperature dependence of the tape. Far from $T_{c}$, the $\mathrm{ReBaCuO}$ resistivity is high, hence, neglecting the buffer layer, the resistance depends on the resistivity of silver and C-276 layers ( [6]- [7]) and their geometrical dimensions (length and cross section). On a SP tape of $847 \mathrm{~mm}$ length, the temperature has been varied from $77 \mathrm{~K}$ up to $350 \mathrm{~K}$ meanwhile, the resistance $(R(T))$ has been monitored using a four wires measurement (blue solid line of figure 2). Given that the length and the width of the sample are known $(847 \mathrm{~mm}$ and $12 \mathrm{~mm})$, it is possible to find out the corresponding thicknesses fitting the $R(T)$ curve. With a thickness of $4.8 \mu \mathrm{m}$ for the silver stabilizer (thAg figure 1c) and a thickness of $104.5 \mu \mathrm{m}$ for the substrate one (thSUB figure $1 c)$, our model fits the measurement with good approximation (dashed black line). Through those easy considerations, it is possible to predict the resistance per unit length given by different silver stabilizations, e.g. $1.8 \mu \mathrm{m}, 2.8 \mu \mathrm{m}$ and $3.8 \mu \mathrm{m}$ (magenta, red and green dashed lines of figure 2). Those parameters have been implemented in our model to carry out the analysis on the inhomogeneity effect.

\section{Critical current tape inhomogeneity representation}

The tape inhomogeneity can be represented by a gaussian distributions, function of the average critical current $\left(I_{c, a v}\right)$ and the standard deviation $(\sigma)$. It is relatively easy to built such distributions from $I_{c}$ measurements based on voltage criterion. The gaussian distribution reported in figure $4\left(I_{c, a v}=365 \mathrm{~A}\right.$ and $\left.\sigma_{\%}=6.59\right)$ is based on experimental data provided us by the courtesy of SuperPower-Inc. The gaussian has been discretized in 20 levels of $I_{c}$ inhomogeneity, hence, arrays $\left[I c_{[1 x 20]}\right]$ and $\left[g_{[1 x 20]}\right]$ have been defined. 

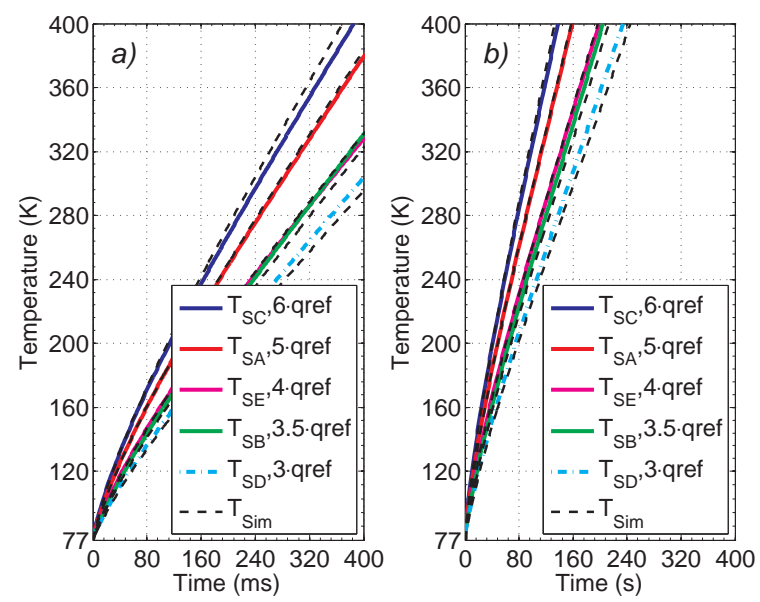

Figure 3: Calibration of $R_{T}$. Fit of the temperature of the first five sectors of the FEM $\left(T_{S A}, T_{S B}, T_{S C}, T_{S D}, T_{S E}\right)$ with the simulink model $\left(T_{\text {sim }}\right)$. Each sectors has a different applied power density, $q_{r e f}=3 W(a)$ and $q_{r e f}=8 W(b)$.

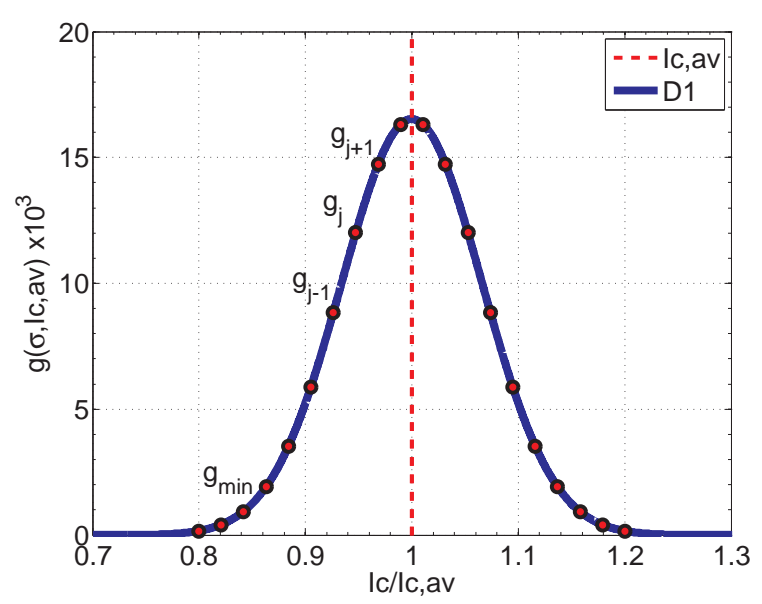

Figure 4: Tape Critical current inhomogeneity. Gaussian distribution D1 has an average critical current of $365 \mathrm{~A}$ and $\sigma \%=6.59$.

A transformation of $\left[g_{[1 \times 20]}\right]$ elements has been done with

$$
P_{j}=\frac{g_{j}}{\sum_{i=1}^{20} g_{i}} \cdot 100
$$

where $P_{j}$ is the probability, in percentage, to find the critical current $I_{c j}$ along the tape length. Finally, a new array $\left[\operatorname{Im}_{[1 x 100]}\right]$ has been built. The elements of $\left[\operatorname{Im}_{[1 x 100]}\right]$ are defined by

$$
\left[\operatorname{Im}_{j}\right]=\left[I c_{j} \cdot \bar{u}_{j}\left(\left[1, \operatorname{int}\left(P_{j}\right)\right]\right)\right]
$$

where $u_{j}$ is an array of length $\operatorname{int}\left(P_{j}\right)$ of unit elements. Since $\sum_{j=1}^{20} P_{j}=100,[\mathrm{Im}]$ has length 100 (corresponding to the number of blocks) and it contains information about critical current levels and their corresponding probability (figure $5 a$ ). The relative position of $[\mathrm{Im}]$ (figure $5 b$ ) elements implies a different amount of thermal conduction between blocks. Therefore, random permutations of $[I m]$ terms have been generated. Array $[\mathrm{Im}]$ and its combinations have been implemented to the electrothermal model.

\section{Influence of the stabilizer thickness}

an RFCL device has been modeled with tapes D1 and it has been studied in the equivalent circuit of figure 6 . Assuming an overcurrent of $20 \% I_{\text {norm }}$ (1 kA RMS), the device can be built with 6 parallel conductors per phase of inhomogeneity D1 (the minimum $I c$ is $0.85 I_{c, a v}$ with $I_{c, a v}=365$ ). The external shunt impedance $\left(Z_{s}=R_{s}+j 2 \pi f L_{s}\right.$, figure 6) determines the limited current allowed [8] and it is normally imposed by utility companies requiring long fault duration. The circuit breaker $(C B 1$ 

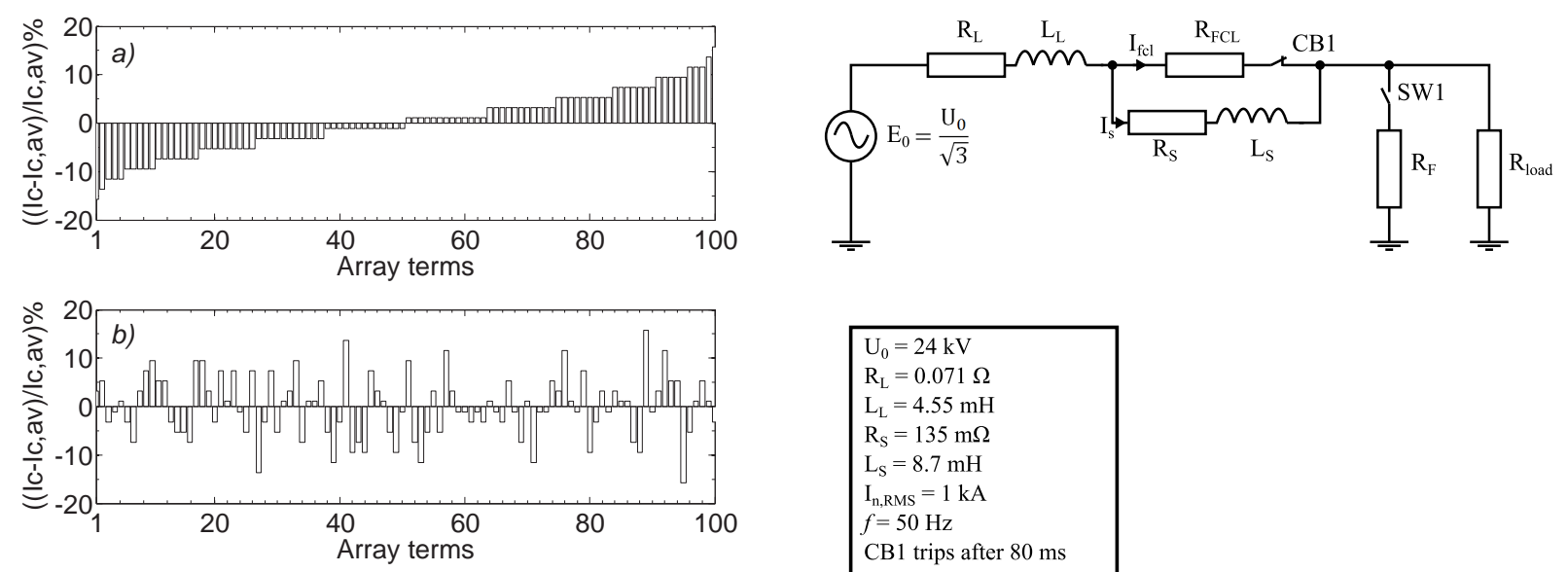

\begin{tabular}{|l|}
\hline $\mathrm{U}_{0}=24 \mathrm{kV}$ \\
$\mathrm{R}_{\mathrm{L}}=0.071 \Omega$ \\
$\mathrm{L}_{\mathrm{L}}=4.55 \mathrm{mH}$ \\
$\mathrm{R}_{\mathrm{S}}=135 \mathrm{~m} \Omega$ \\
$\mathrm{L}_{\mathrm{S}}=8.7 \mathrm{mH}$ \\
$\mathrm{I}_{\mathrm{n}, \mathrm{RMS}}=1 \mathrm{kA}$ \\
$f=50 \mathrm{~Hz}$ \\
$\mathrm{CB} 1$ trips after $80 \mathrm{~ms}$ \\
SW1 switches after $20 \mathrm{~ms}$ \\
\hline
\end{tabular}

Figure 5: Gaussian distribution discretization. Base array $[\mathrm{Im}]$ of distribution D1 $(a)$ and a random combination of the base array $(b)$.

Figure 6: Equivalent circuit. The resistance $R_{F}$ has been varied from 0 (clear three phases short circuit fault) up to $30 \Omega$.

figure 6) tripping time $\left(t_{C B}\right)$ is about $80 \mathrm{~ms}$. The thickness of the silver stabilizer has been varied from $1.8 \mu \mathrm{m}$ up to $4.8 \mu \mathrm{m}$. For each value, the single conductor length has been chosen in order to respect the maximum allowable temperature criteria of 360 $K$ under clear three phases short-circuit (fault resistance $R_{F}=0 \Omega$ ). Even though it corresponds to optimize the device against the maximum energy assorted in the circuit, this approach does not allow to exclude thermal instability of the RFCL under low values of fault current $\left(R_{F}>>0\right)$. Therefore, a correct analysis of the thickness stabilizer should consider the whole fault current range. Since we do not consider differences in inhomogeneity between the paralleled tapes per phases, the following dissertation is refereed to a single wire.

\subsection{With $R_{F} \simeq \boldsymbol{O}$.}

Under this condition, the current through each tape is much higher then $I_{c, a v}$. The transition to normal state is extremely rapid and homogeneous (all zones quench in the same instant). The RFCL offers to the circuit its highest impedance. During the limiting performance the SC material is stressed $\left(I_{s c}>I c, a v\right)$ for few $m s$ (red line inset of figure 7) and almost all the fault current is diverted to the external shunt (blue line figure 7). A tape with thin stabilizer has an high resistance per unit length $\left(R_{\text {p.u.l. }}\right)$. Therefore, for a given value of $Z_{s}$ and $t_{C B}$, it allows to face the temperature criterion $(360 \mathrm{~K})$ minimizing the lengths of the paralleled wires with evident benefits of the final cost of the RFCL device.

\subsection{With $R_{F}>>0$.}

This subsection analyzes the influence of the wire stabilization on the transient device performance in case of high value of fault resistance (low value of fault current). 


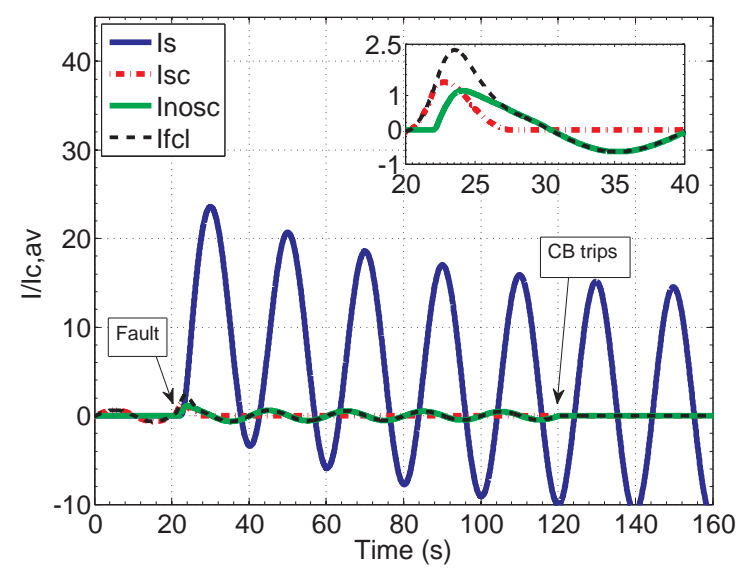

Figure 7: Current sharing between external shunt $\left(I_{s}\right)$, SC layer $\left(I_{s c}\right)$ and no superconducting layers $\left(I_{\text {nosc }}\right)$ with tape inhomogeneity $\mathrm{D} 1, \mathrm{Zs}=2.7 \Omega$ and $R_{F}=0$. The sc layer is stressed only for few $m s$ (inset).
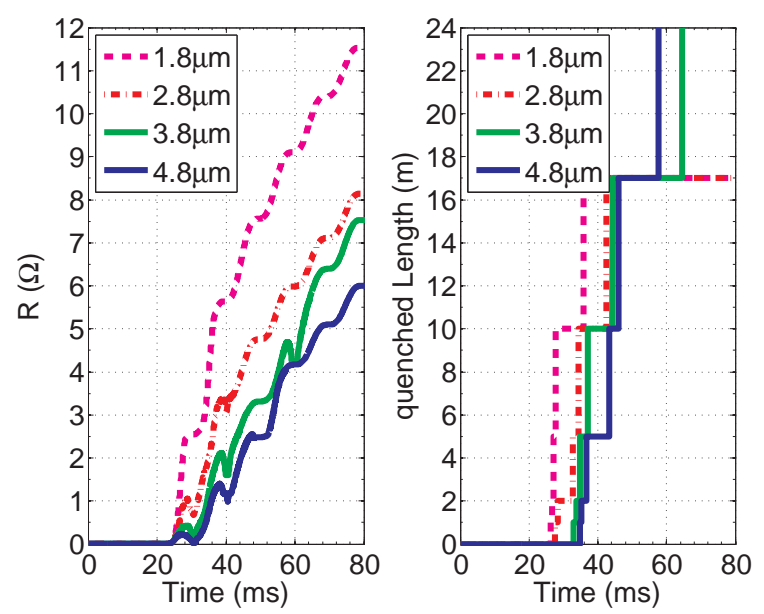

Figure 8: Resistance profile of the device (a) and quenched length of a single CC (b) for different silver stabilizer thicknesses under $R_{F}=14 \Omega$.

6.2.1. Low stabilization. Even if $I_{F}$ is around $I_{c, t o t}$, due to the tape inhomogeneity, the zones with low critical current $\left(I_{c}<0.9 I_{c, a v}\right)$ may quench and limit the current through the RFCL device. In case of low silver stabilization (e.g. $1.8 \mu \mathrm{m}$ ), those zones will introduce a high resistance (figure $8 a$ magenta line) and $I_{F}$ may suddenly fall below $I_{c, a v}$. As consequence, zones with higher $I_{c}(I c>0.9 I c, a v)$ may not have enough energy to quench $\left(I_{F}\right.$ flows through the SC layer, figure $\left.9 a\right)$. All the current is limited by a small portion (limiting length, $L_{l i m}$ ) of the tape (figure $8 b$ ). The temperature increase is manly hindered by the heat capacity of the quenched tape portion. Therefore if this portion is too "short", some zones of $L_{\text {lim }}$ could exceed $360 \mathrm{~K}$ before the intervention of $C B 1$ (80 $\mathrm{ms}$ in our example).

6.2.2. High stabilization. Since the silver stabilization of the tape do not influence the critical current inhomogeneity, even with a more stabilized wire (e.g $4.8 \mu \mathrm{m}$ ), zones with low critical current $(I c<0.9 I c, a v)$ will quench. Differently from the previous case, those zones will offer a lower resistance (figure $8 a$ blue line), hence, after the first peak an higher current will flow through the RFCL device (black dashed line figure $9 b$ ). More energy will be provided to zones with $I_{c} \simeq I_{c, a v}$ ( $I_{F} \simeq I_{c, a v}$ for longer time) that will quench. Compared to the previous case, $L_{\text {lim }}$ is longer and so an higher heat capacity contributes to the limiting performance. Figure 10 shows clearly that when $R_{F} \simeq 0$, adjusting the single tape length, it is possible to respect the temperature criteria in all cases. On the contrary, extending the analysis to $R_{F}>>0$ stabilizer thicknesses of $1.8 \mu \mathrm{m}$ and $2.8 \mu \mathrm{m}$ "leave" a dangerous range of possible fault currents (blue and red lines), whereas an higher stabilization $(3.8 \mu \mathrm{m}$ and $4.8 \mu \mathrm{m})$ guarantees thermal stability against the whole range of fault currents. 

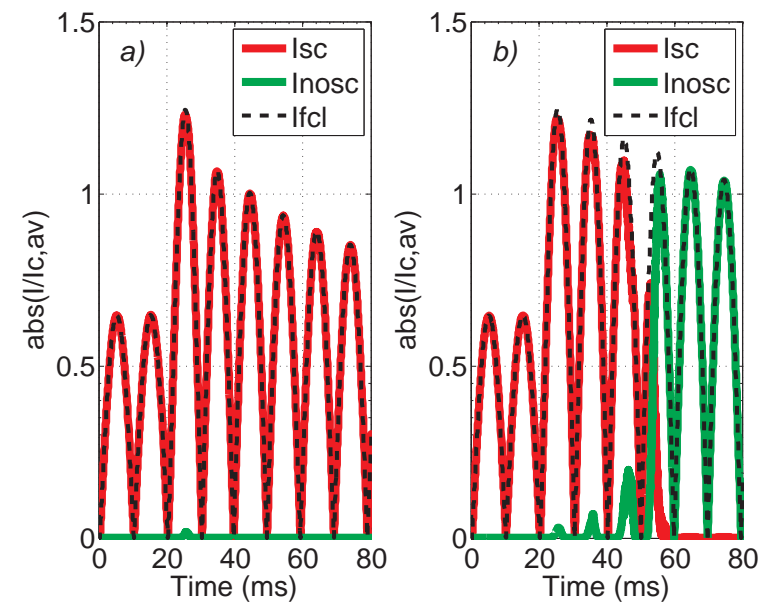

Figure 9: Current sharing between SC and noSC layers in blocks having $I c_{k} / I c, a v=0.95$ with silver stabilizer thickness of $1.8 \mu m(a)$ and $4.8 \mu \mathrm{m}(b)$ when the inhomogeneity is D1 and $R_{F}=14 \Omega$.

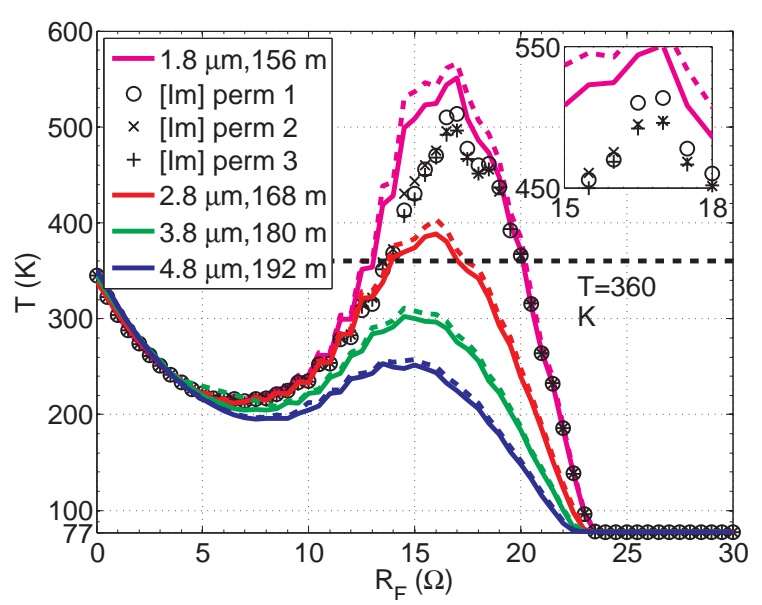

Figure 10: Final temperature foreseen for different stabilizer thicknesses. Base $[\mathrm{Im}]$ array (solid lines), without thermal conduction (dashed lines) and three random permutations of $[\mathrm{Im}]$ terms (only for thAg=1.8 $\mu \mathrm{m}$ ).

\subsection{Consideration about thermal model validity}

With low value of $R_{F}$ (high value of fault current, $I_{F}$ ), the quench tends to be homogeneous. The heat exchanged between different zones of the tape is low, hence, neglecting the thermal conduction represents a good approximation (dashed lines figure 10) that does not affect significantly the final result. In the other hand, increasing $R_{F}$ (low value of fault current), the conduction starts to be significant and the maximum temperature foreseen $\left(T_{\max }\right)$ differs from the one obtained without conduction between blocks. Since the thermal conduction is proportional to the temperature difference of the contiguous blocks, $[\mathrm{Im}]$ gives the minimum $T_{\max }$ possible (solid lines figure 10). Any other combination of $[\mathrm{Im}]$ terms leads to a lower $T_{\max }$ (the inset of figure 10 shows only the case of th $\mathrm{Ag}=1.8 \mu \mathrm{m})$. In conclusion, neglecting the conduction between blocks is the most conservative approach and in general it tends to an overestimation of the $T_{\max }$. In any case our method allows a more precise calculation of the device final temperature.

\section{Conclusion}

This paper analyzes the inhomogeneity effects on the limiting performance of RFCLs for MV applications. We developed a numerical model in SimPowerSystem ${ }^{\mathrm{TM}}$ that allows to evaluate inhomogeneous HTS-CC candidates, optimize their stabilization and study the FCL behaviors once inserted in the electricity network. Our approach can consider the thermal conduction within the tape in conditions where is not possible to neglect it. The geometrical manufacturing imperfections of silver stabilizer and hastelloy substrate can be considered analyzing $R(T)$ measurements. A dynamical calibration of the thermal part of the model has been performed using finite element method. Since 
under high values of prospective current $\left(I_{F}>>I_{c, a v}\right)$ there is homogenous quench, for any thickness silver stabilizer it is possible to obtain the desired degree of limitation adjusting the length of the tapes. It seems obvious to prefer tapes with high $R_{\text {p.u.l. }}$ (low thickness of silver stabilizer). However, the analysis of low fault currents leads to different conclusions. Under $I_{F} \simeq I_{c, \text { min }}$, due to $I_{c}$ inhomogeneity, the quench tends to be partial. The length of the tape that contribute to the transient limitation is inversely proportional to the silver stabilizer thickness. If the limiting zone is too small, the high power density concentration may brings to dangerous values of temperature. A thicker silver stabilizer allows to have a less sharp limitation of $I_{F}$ spreading the power dissipation on a longer lengths of tape. This permits to reduce the temperature increase and to guarantee thermal stability against the whole range of fault currents the grid can present.

\section{Acknowledgements}

This work was partly supported by the European Union Seventh Framework Programme (FP7/2007-2013). Grant Agreement NO.241285. "ENERGY.2009.7.3.1: HTS Devices for electricity networks" (ECCOFLOW) [9]. The authors would like to thank SuperPower Inc. for providing the sample data used in this work.

\section{References}

[1] C. Neumann, 2006, IEEE Power Engineering Society General Meeting, PES, art. no. 1709336.

[2] SuperPower Inc. [Online]. http://www.superpower-inc.com.

[3] D. K. Park et al, 2009, Cryogenics, 49, no. 6, 249-253.

[4] R. Lortz et al, 2006, Physica C, 434, 194-198.

[5] D. Colangelo et al, presented at the EUCAS 2011, The Hague, The Netherlands, Sep. 1823, 2011, in press.

[6] Handbook of Chemistry and Physics, 12-42, [Online]. http://www.hbcpnetbase.com/

[7] J. Lu, E. S. Choi, and H. D. Zhou, 2008, J. Appl. Phys. 103, 064908 ; doi: 10.1063/1.2899058

[8] P. Tixador and N. T. Nguyen, 2012, Supercond. Sci. Technol., 25, 014009.

[9] Eccoflow website [Online]. http://www.eccoflow.org. 